\title{
The geometry of product conjugate connections
}

\author{
Adara M. Blaga and Mircea Crasmareanu \\ Dedicated to Professor Vasile Cruceanu on the occasion of his 80th birthday \\ An. Stiint. Univ. Al. I. Cuza Iasi Math., 59(2013), no. 1, 73-84
}

\begin{abstract}
Properties of pairs of product conjugate connections are stated with a special view towards the integrability of the given almost product structure. We define the analogous in product geometry of the structural and the virtual tensors from the Hermitian geometry and express the product conjugate connections in terms of these tensors. Some examples from the geometry of a pair of complementary distributions are discussed and for this case the above structural and virtual tensors are expressed in terms of O'Neill-Gray tensor fields.
\end{abstract}

AMS Subject Classification: 53B05, 53C15.

Keywords and phrases: almost product structure; (conjugate) linear connection; almost product Riemannian manifold; structural and virtual tensor field.

\section{Introduction}

Fix $M$ a smooth, $n$-dimensional manifold for which we denote: $C^{\infty}(M)-$ the algebra of smooth real functions on $M, \mathfrak{X}(M)$ - the Lie algebra of vector fields on $M, T_{s}^{r}(M)$ - the $C^{\infty}(M)$-module of tensor fields of $(r, s)$-type on $M$. Usually $X, Y, Z, \ldots$ will be vector fields on $M$ and if $T \rightarrow M$ is a vector bundle over $M$, then $\Gamma(T)$ denotes the $C^{\infty}$-module of sections of $T$ [e.g. $\Gamma(T M)=\mathfrak{X}(M)]$.

Let $\mathcal{C}(M)$ be the set of linear connections on $M$. Since the difference of two linear connections is a tensor field of $(1,2)$-type, it results that $\mathcal{C}(M)$ is a $C^{\infty}(M)$-affine module associated to the $C^{\infty}(M)$-linear module $T_{2}^{1}(M)$.

Fix now $E$ an almost product structure on $M$, i.e. an endomorphism of the tangent bundle such that $E^{2}=I_{\mathfrak{X}(M)}$. Then the associated linear connections are: 
Definition 0.1 $\nabla \in \mathcal{C}(M)$ is an E-connection if $E$ is covariant constant with respect to $\nabla$, namely $\nabla E=0$. Let $\mathcal{C}_{E}(M)$ be the set of these connections.

In order to find the above set, let us consider after [5, p. 342] the maps

$$
\psi_{E}: \mathcal{C}(M) \rightarrow \mathcal{C}(M), \quad \chi_{E}: T_{2}^{1}(M) \rightarrow T_{2}^{1}(M)
$$

given by

$$
\psi_{E}(\nabla):=\frac{1}{2}(\nabla+E \circ \nabla \circ E), \quad \chi_{E}(\tau):=\frac{1}{2}(\tau+E \circ \tau \circ E) .
$$

So

$$
\left\{\begin{array}{l}
\psi_{E}(\nabla)_{X} Y=\frac{1}{2}\left[\nabla_{X} Y+E\left(\nabla_{X} E Y\right)\right] \\
\chi_{E}(\tau)(X, Y)=\frac{1}{2}[\tau(X, Y)+E(\tau(X, E Y))] .
\end{array}\right.
$$

Then, $\psi_{E}$ is a $C^{\infty}(M)$-projector on $\mathcal{C}(M)$ associated to the $C^{\infty}(M)$-linear projector $\chi_{E}$ :

$$
\psi_{E}^{2}=\psi_{E}, \quad \chi_{E}^{2}=\chi_{E}, \quad \psi_{E}(\nabla+\tau)=\psi_{E}(\nabla)+\chi_{E}(\tau) .
$$

It follows that $\nabla E=0$ means $\psi_{E}(\nabla)=\nabla$ which gives that $\mathcal{C}_{E}(M)=$ $\operatorname{Im} \psi_{E}$. This determines completely $\mathcal{C}_{E}(M)$. Fix $\nabla_{0}$ arbitrary in $\mathcal{C}(M)$ and $\nabla$ in $\mathcal{C}_{E}(M)$. So, $\nabla=\psi_{E}\left(\nabla^{\prime}\right)$ with $\nabla^{\prime}=\nabla_{0}+\tau$. In conclusion, $\nabla=\psi_{E}\left(\nabla_{0}\right)+\chi_{E}(\tau)$; in other words, $\mathcal{C}_{E}(M)$ is the affine submodule of $\mathcal{C}(M)$ passing through the $E$-connection $\psi_{E}\left(\nabla_{0}\right)$ and having the direction given by the linear submodule $\operatorname{Im} \chi_{E}$ of $T_{2}^{1}(M)$.

Let us remark a decomposition (of arithmetic mean type) of it [5, p. 343]:

$$
\psi_{E}(\nabla)=\frac{1}{2}\left(\nabla+C_{E}(\nabla)\right)
$$

with the conjugation map $C_{E}: \mathcal{C}(M) \rightarrow \mathcal{C}(M)$ :

$$
C_{E}(\nabla)_{X}:=E \circ \nabla_{X} \circ E .
$$

Then the product conjugate connection $C_{E}(\nabla)$ measures how far the connection $\nabla$ is from being an $E$-connection and as it is pointed out in [5, p. 343], $C_{E}$ is the affine symmetry of the affine module $\mathcal{C}(M)$ with respect to the affine submodule $\mathcal{C}_{E}(M)$, made parallel with the linear submodule ker $\chi_{E}$.

The present paper is devoted to a large study of this new connection $C_{E}(\nabla)$, since all above computations put in evidence its rôle in the geometry 
of $E$. Therefore, the aim of our study is to obtain several properties of it in both the general case and Riemannian geometry. The first section is devoted to this scope and after a general result connecting $\nabla$ and $C_{E}(\nabla)$, we treat two items:

i) the behavior of the product conjugate connections to a linear change of almost product structure,

ii) the introduction in the product geometry of two tensor fields previously considered in the complex geometry.

With respect to i) we arrive at two particular remarkable cases concerning the recurrence of the given almost product structures, while for ii) we derive some useful new identities.

The second part of this paper is directed towards examples and to put in our framework the geometry of (two complementary) distributions. The most important case is when the considered distributions are in a natural relationship with the initial linear connection $\nabla$ or with the almost product structure $E$.

In the last section we give some generalizations of the results from the first part by adding an arbitrary tensor field of (1,2)-type. All generalized product conjugate connections which form a duality with the initial linear connection are determined.

\section{Properties of the product conjugate connection}

In what follows, for simplification we will denote by a superscript $E$ the product conjugate connection of $\nabla$

$$
\nabla^{(E)}:=C_{E}(\nabla)=\nabla+E \circ \nabla E
$$

and then

$$
\nabla_{X}^{(E)} Y=\nabla_{X} Y+E\left(\nabla_{X} E Y-E\left(\nabla_{X} Y\right)\right)=E\left(\nabla_{X} E Y\right)
$$

The first properties of the product conjugate connection are stated in the next proposition:

Proposition 1.1 Let $E$ be an almost product structure, $\nabla$ a linear connection and $\nabla^{(E)}$ the product conjugate connection of $\nabla$. Then:

1. $\nabla^{(E)} E=-\nabla E$; it results that $\nabla \in \mathcal{C}_{E}(M)$ if and only if $\nabla^{(E)} \in$ $\mathcal{C}_{E}(M)$. 
2. $\nabla$ and $\nabla^{(E)}$ are in duality: $\left(\nabla^{(E)}\right)^{(E)}=\nabla$.

3. $T_{\nabla_{(E)}}=T_{\nabla}+E\left(d^{\nabla} E\right)$, where $d^{\nabla}$ is the exterior covariant derivative induced by $\nabla$, namely $\left(d^{\nabla} E\right)(X, Y):=\left(\nabla_{X} E\right) Y-\left(\nabla_{Y} E\right) X$; it results that for $\nabla \in \mathcal{C}_{E}(M)$, the connections $\nabla$ and $\nabla^{(E)}$ have the same torsion.

4. $R_{\nabla^{(E)}}(X, Y, Z)=E\left(R_{\nabla}(X, Y, E Z)\right)$; it results that $\nabla$ is flat if and only if $\nabla^{(E)}$ is so.

5. Assume that $(M, g, E)$ is an almost product Riemannian manifold, i.e. $g(E X, E Y)=g(X, Y)$. Then $\left(\nabla_{X}^{(E)} g\right)(E Y, E Z)=\left(\nabla_{X} g\right)(Y, Z)$; it results that $\nabla$ is a $g$-metric connection if and only if $\nabla^{(E)}$ is so.

Proof 1 . The main relations used here are

$$
\nabla_{X}^{(E)} E Y=E\left(\nabla_{X} Y\right), \quad E\left(\nabla_{X}^{(E)} Y\right)=\nabla_{X} E Y
$$

and then

$$
\left(\nabla_{X} E\right) Y=\nabla_{X} E Y-E\left(\nabla_{X} Y\right)=E\left(\nabla_{X}^{(E)} Y\right)-\nabla_{X}^{(E)} E Y=-\left(\nabla_{X}^{(E)} E\right) Y .
$$

2. Although a direct proof can be provided by the formula (0.6), we prefer a proof here, in order to use (1.1):

$$
\left(\nabla^{(E)}\right)^{(E)}=\nabla^{(E)}+E \circ \nabla^{(E)} E=\nabla+E \circ \nabla E+E \circ(-\nabla E)=\nabla .
$$

3. A direct computation gives

$$
\begin{gathered}
T_{\nabla^{(E)}}(X, Y):=\nabla_{X}^{(E)} Y-\nabla_{Y}^{(E)} X-[X, Y]=E\left(\nabla_{X} E Y\right)-E\left(\nabla_{Y} E X\right)-[X, Y]= \\
=E\left(\nabla_{X} E Y-\nabla_{Y} E X\right)+T_{\nabla}(X, Y)-\nabla_{X} Y+\nabla_{Y} X:= \\
:=T_{\nabla}(X, Y)+E\left(\left(\nabla_{X} E\right) Y-\left(\nabla_{Y} E\right) X\right) .
\end{gathered}
$$

4.

$$
\begin{gathered}
R_{\nabla^{(E)}}(X, Y, Z):=\nabla_{X}^{(E)} \nabla_{Y}^{(E)} Z-\nabla_{Y}^{(E)} \nabla_{X}^{(E)} Z-\nabla_{[X, Y]}^{(E)} Z= \\
=\nabla_{X}^{(E)} E\left(\nabla_{Y} E Z\right)-\nabla_{Y}^{(E)} E\left(\nabla_{X} E Z\right)-E\left(\nabla_{[X, Y]} E Z\right)= \\
=E\left(\nabla_{X} \nabla_{Y} E Z\right)-E\left(\nabla_{Y} \nabla_{X} E Z\right)-E\left(\nabla_{[X, Y]} E Z\right):=E\left(R_{\nabla}(X, Y, E Z)\right) .
\end{gathered}
$$

5.

$$
\left(\nabla_{X}^{(E)} g\right)(V, W):=X(g(V, W))-g\left(\nabla_{X}^{(E)} V, W\right)-g\left(V, \nabla_{X}^{(E)} W\right)=
$$




$$
=X(g(V, W))-g\left(E\left(\nabla_{X} E V\right), W\right)-g\left(V, E\left(\nabla_{X} E W\right)\right)
$$

for any $X, V$ and $W \in \mathfrak{X}(M)$. With $V:=E Y$ and $W:=E Z$, we get

$$
\begin{gathered}
\left(\nabla_{X}^{(E)} g\right)(E Y, E Z)=X(g(E Y, E Z))-g\left(E\left(\nabla_{X} Y\right), E Z\right)-g\left(E Y, E\left(\nabla_{X} Z\right)\right)= \\
=X(g(Y, Z))-g\left(\nabla_{X} Y, Z\right)-g\left(Y, \nabla_{X} Z\right):=\left(\nabla_{X} g\right)(Y, Z) .
\end{gathered}
$$

The above substitutions hold for $Y=E V$ and $Z=E W$.

There are some direct consequences of these formulae:

i) if $\nabla$ is the Levi-Civita connection of $g$, then $\nabla^{(E)}$ is also metric with respect to $g$,

ii) if $\nabla$ is the Levi-Civita connection of $g$ and in addition $\nabla \in \mathcal{C}_{E}(M)$, then $\nabla^{(E)}=\nabla$ as the unique symmetric $g$-metric connection.

More generally, let $f \in \operatorname{Dif} f(M)$ be an automorphism of the $G$-structure defined by $E$, i.e. $f_{*} \circ E=E \circ f_{*}$. If $f$ is an affine transformation for $\nabla$, namely $f_{*}\left(\nabla_{X} Y\right)=\nabla_{f_{*} X} f_{*} Y$, then $f$ is also affine transformation for $\nabla^{(E)}$.

Two natural generalizations of the case $\nabla \in \mathcal{C}_{E}(M)$ are given by:

Proposition 1.2 Let $\nabla$ be a symmetric linear connection.

i) Assume that $E$ is $\nabla$-recurrent, i.e. $\nabla E=\eta \otimes E$, where $\eta$ is a 1-form. Then $\nabla^{(E)}$ is a semi-symmetric connection.

ii) Assume that $\nabla E=\eta \otimes I_{\mathfrak{X}(M)}$. Then $\nabla^{(E)}$ is a quarter-symmetric connection.

Proof i) We have $\nabla^{(E)}=\nabla+\eta \otimes I$ and from the item 3 of the previous Proposition, we get $T_{\nabla(E)}=\eta \otimes I-I \otimes \eta$.

ii) It results that $\nabla^{(E)}=\nabla+\eta \otimes E$ and, as above, we get $T_{\nabla^{(E)}}=\eta \otimes E-E \otimes \eta$.

The next subject consists of the behavior of $\nabla^{(\cdot)}$ for families of almost product structures. Let $E_{1}$ and $E_{2}$ be two almost product structures and consider the pencil of $(1,1)$-tensor fields $E_{\alpha, \beta}:=\alpha E_{1}+\beta E_{2}$ with $\alpha$ and $\beta \in \mathbb{R}$. In order that $E_{\alpha, \beta}$ to be an almost product structure there are necessary two conditions:

1) $E_{1}$ and $E_{2}$ be skew-commuting structures: $E_{1} E_{2}=-E_{2} E_{1}$,

2) $(\alpha, \beta)$ belongs to the unit circle $S^{1}: \alpha^{2}+\beta^{2}=1$.

Then:

$$
\nabla_{X}^{\left(E_{\alpha, \beta}\right)} Y=\alpha^{2} \nabla_{X}^{\left(E_{1}\right)} Y+\beta^{2} \nabla_{X}^{\left(E_{2}\right)} Y+\alpha \beta\left[E_{1}\left(\nabla_{X} E_{2} Y\right)+E_{2}\left(\nabla_{X} E_{1} Y\right)\right]
$$

and there can be distinguished two remarkable particular cases:

i) if $E_{1}$ and $E_{2}$ are recurrent with respect to $\nabla$ with the same 1-form of 
recurrence: $\nabla E_{i}=\eta \otimes E_{i}$, then the product conjugate connections coincide $\nabla^{\left(E_{1}\right)} \equiv \nabla^{\left(E_{2}\right)}=: \nabla^{\left(E_{12}\right)}$ and it follows the invariance of $\nabla^{(E)}$ :

$$
\nabla^{\left(E_{\alpha, \beta}\right)}=\nabla^{\left(E_{12}\right)}
$$

ii) assume that the triple $\left(\nabla, E_{1}, E_{2}\right)$ is a mixed-recurrent structure: $\nabla E_{i}=$ $\eta \otimes E_{j}$ with $i \neq j$. Then $\nabla$ is the average of the two product conjugate connections, $\nabla=\frac{1}{2}\left(\nabla^{\left(E_{1}\right)}+\nabla^{\left(E_{2}\right)}\right)$ and

$$
\nabla^{\left(E_{\alpha, \beta}\right)}=\nabla+\left(\alpha^{2}-\beta^{2}\right) \eta \otimes E_{1} E_{2} .
$$

The last subject of this section treats two tensor fields associated to an almost product structure. The paper [7] introduces the structural and virtual tensor fields of an almost complex structure. Turning into our framework, let us consider for a pair $(\nabla, E)$ the tensor fields of $(1,2)$-type:

1) the structural tensor field

$$
C_{\nabla}^{E}(X, Y):=\frac{1}{2}\left[\left(\nabla_{E X} E\right) Y+\left(\nabla_{X} E\right) E Y\right]
$$

2) the virtual tensor field

$$
B_{\nabla}^{E}(X, Y):=\frac{1}{2}\left[\left(\nabla_{E X} E\right) Y-\left(\nabla_{X} E\right) E Y\right]
$$

From the item 1 of the first Proposition it results that both these tensor fields are skew-symmetric with respect to the product conjugation of connections:

$$
C_{\nabla^{(E)}}^{E}=-C_{\nabla}^{E}, \quad B_{\nabla^{(E)}}^{E}=-B_{\nabla}^{E} .
$$

Also

$$
C_{\nabla}^{E}(E X, E Y)=C_{\nabla}^{E}(X, Y), B_{\nabla}^{E}(E X, E Y)=-B_{\nabla}^{E}(X, Y) .
$$

The importance of these tensor fields for our study is given by the following straightforward relation:

$$
\nabla^{(E)}=\nabla-C_{\nabla}^{E}+B_{\nabla}^{E}
$$

Recall after [4] that two linear connections are called projectively equivalent if there exists a 1-form $\tau$ such that:

$$
\nabla^{\prime}=\nabla+\tau \otimes I+I \otimes \tau .
$$

A straightforward calculus gives that $C^{E}$ is invariant for projectively changes (1.16) while for $B^{E}$ we have:

$$
\left(B_{\nabla^{\prime}}^{E}-B_{\nabla}^{E}\right)(X, Y)=\tau(E Y) E X-\tau(Y) X .
$$


The geometry of product conjugate connections

\section{Invariant distributions}

Let $\mathcal{D} \subset T M$ be a fixed distribution considered as a vector subbundle of $T M$.

Definition 2.1 i) $\mathcal{D}$ is called $E$-invariant if $X \in \Gamma(\mathcal{D})$ implies $E X \in$ $\Gamma(\mathcal{D})$.

ii) ([3, p. 210]) The linear connection $\nabla$ restricts to $\mathcal{D}$ if $Y \in \Gamma(\mathcal{D})$ implies $\nabla_{X} Y \in \Gamma(\mathcal{D})$, for any $X \in \Gamma(T M)$.

If $\nabla$ restricts to $\mathcal{D}$, then $\nabla$ may be considered as a connection in the vector bundle $\mathcal{D}$. From this fact, in [1, p. 7] a connection which restricts to $\mathcal{D}$ is called adapted to $\mathcal{D}$.

Proposition 2.2 If the distribution $\mathcal{D}$ is E-invariant and the linear connection $\nabla$ restricts to $\mathcal{D}$, then $\nabla^{(E)}$ also restricts to $\mathcal{D}$.

Proof Fix $Y \in \Gamma(\mathcal{D})$. Then $E Y \in \Gamma(\mathcal{D})$ and for any $X \in \Gamma(T M)$ we have $\nabla_{X} Y \in \Gamma(\mathcal{D})$. Therefore, $\nabla_{X}^{(E)} Y=E\left(\nabla_{X} E Y\right) \in \Gamma(\mathcal{D})$.

A more general notion like restricting to a distribution is that of geodesically invariance [3, p. 118]. The distribution $\mathcal{D}$ is $\nabla$-geodesically invariant if for every geodesic $\gamma:[a, b] \rightarrow M$ of $\nabla$ with $\dot{\gamma}(a) \in \mathcal{D}_{\gamma(a)}$ it follows $\dot{\gamma}(t) \in \mathcal{D}_{\gamma(t)}$ for any $t \in[a, b]$. The cited book gives a necessary and sufficient condition for a distribution $\mathcal{D}$ to be $\nabla$-geodesically invariant: for any $X$ and $Y \in \Gamma(\mathcal{D})$, the symmetric product $\langle X: Y\rangle:=\nabla_{X} Y+\nabla_{Y} X$ to belong to $\Gamma(\mathcal{D})$ or equivalently, for any $X \in \Gamma(\mathcal{D})$ to have $\nabla_{X} X \in \Gamma(\mathcal{D})$.

The following result is a direct consequence of definitions:

Proposition 2.3 If the distribution $\mathcal{D}$ is E-invariant and the linear connection $\nabla$ restricts to $\mathcal{D}$, then $\mathcal{D}$ is geodesically invariant for $\nabla^{(E)}$.

Example 2.4 Assume that the tangent bundle $T M$ admits a decomposition

$$
T M=V \oplus H
$$

into vertical and horizontal vectors. Let $\mathcal{D}_{v}=\Gamma(V)$ respectively, $\mathcal{D}_{h}=\Gamma(H)$ and the corresponding projectors $v$ and $h$. Then $E=h-v$ is an almost product structure and both $\mathcal{D}_{*}$ are $E$-invariant. As it is proved in [1], the almost product structures are in a natural relationship with decompositions of (2.1)-type and so, $E=h-v$ is the prototype of all possible almost product structures.

The product conjugate connection of $\nabla$ is

$$
\nabla_{X}^{(E)} Y=h\left(\nabla_{X} h Y\right)-h\left(\nabla_{X} v Y\right)-v\left(\nabla_{X} h Y\right)+v\left(\nabla_{X} v Y\right)
$$


and then we have:

Proposition 2.5 If $\nabla^{(E)}$ is torsion-free, then $E$ is integrable, which means that $\mathcal{D}_{h}$ and $\mathcal{D}_{v}$ are involutive distributions.

Proof From (2.2) we get

$h[X, Y]+v[X, Y]=\nabla_{X}^{(E)} Y-\nabla_{Y}^{(E)} X=h\left(\nabla_{X} h Y-\nabla_{Y} h X\right)+v\left(\nabla_{X} v Y-\nabla_{Y} v X\right)$

and then

$$
h[X, Y]=\nabla_{X} h Y-\nabla_{Y} h X, \quad v[X, Y]=\nabla_{X} v Y-\nabla_{Y} v X .
$$

With $X \rightarrow v X$ and $Y \rightarrow v Y$ in the first relation above it follows $h[v X, v Y]=$ 0 and the change $X \rightarrow h X$ and $Y \rightarrow v Y$ in the second relation yields $v[h X, h Y]=0$.

We have:

1) $\nabla$ restricts to $\mathcal{D}_{h}$ means $v\left(\nabla_{X} h Y\right)=0$ and $h\left(\nabla_{X} h Y\right)=\nabla_{X} h Y$,

2) $\nabla$ restricts to $\mathcal{D}_{v}$ means $h\left(\nabla_{X} v Y\right)=0$ and $v\left(\nabla_{X} v Y\right)=\nabla_{X} v Y$.

A straightforward computation gives that the general $\nabla^{(E)}$ of $(2.2)$ restricts to $\mathcal{D}_{h}$ and $\mathcal{D}_{v}$. Moreover, if $\nabla$ restricts to both $\mathcal{D}_{*}$, then

$$
\nabla_{X}^{(E)} Y=\nabla_{X} h Y+\nabla_{X} v Y=\nabla_{X} Y
$$

and so $\nabla \in \mathcal{C}_{E}(M)$. Let us remark that the above connection (2.3) is exactly the Schouten connection of the pair $(h, v)$ [6, p. 10]:

$$
\nabla_{X} Y=h\left(\nabla_{X} h Y\right)+v\left(\nabla_{X} v Y\right) .
$$

Example 2.5 Let $M$ be a vector bundle over the manifold $U$ by $\pi$ : $M \rightarrow U$ and $V=\operatorname{ker} T \pi$ the vertical bundle over $M$. Let also $H_{1}$ and $H_{2}$ be two horizontal bundles in the decomposition (2.1) and denote $h_{1}$ respectively, $h_{2}$ their projectors. Then the skew-symmetry $E_{1} E_{2}=-E_{2} E_{1}$ for the corresponding almost product structures of the previous example means the skew-symmetry $h_{1} h_{2}=-h_{2} h_{1}$.

Sometimes, a complementary distribution $H$ to the above vertical subbundle $V$ is called Ehresmann connection, while if $M$ is exactly the tangent bundle $T U$, then $H$ is called nonlinear connection [2].

Example 2.6 Let $E$ be the almost product structure of example 2.4. Our next step is to express the Kirichenko tensor fields in terms of the projectors $h, v$ : 
Proposition 2.7 The structural and virtual tensor fields of $E=h-v$ are:

$$
\left\{\begin{array}{l}
C_{\nabla}^{h-v}(X, Y)=2\left[h\left(\nabla_{v X} v Y\right)+v\left(\nabla_{h X} h Y\right)\right] \\
B_{\nabla}^{h-v}(X, Y)=-2\left[h\left(\nabla_{h X} v Y\right)+v\left(\nabla_{v X} h Y\right)\right] .
\end{array}\right.
$$

Proof From (1.14) we get

$$
\left\{\begin{array}{l}
C_{\nabla}^{h-v}(h X, v Y)=-C_{\nabla}^{h-v}(v X, h Y) \\
B_{\nabla}^{h-v}(h X, h Y)=-B_{\nabla}^{h-v}(v X, v Y) .
\end{array}\right.
$$

By making $X \rightarrow v X$ in the first relation and $X \rightarrow h X$ in the second one, it results

$$
\left\{\begin{array}{l}
C_{\nabla}^{h-v}(h X, v Y)=0=C_{\nabla}^{h-v}(v X, h Y) \\
B_{\nabla}^{h-v}(h X, h Y)=0=B_{\nabla}^{h-v}(v X, v Y)
\end{array}\right.
$$

and then

$$
\left\{\begin{array}{l}
C_{\nabla}^{h-v}(X, Y)=C_{\nabla}^{h-v}(h X, h Y)+C_{\nabla}^{h-v}(v X, v Y) \\
B_{\nabla}^{h-v}(X, Y)=B_{\nabla}^{h-v}(h X, h Y)+B_{\nabla}^{h-v}(v X, v Y) .
\end{array}\right.
$$

But

$$
\left\{\begin{array}{l}
C_{\nabla}^{h-v}(h X, h Y)=2 v\left(\nabla_{h X} h Y\right) \\
C_{\nabla}^{h-v}(v X, v Y)=2 h\left(\nabla_{v X} v Y\right)
\end{array}\right.
$$

and

$$
\left\{\begin{array}{l}
B_{\nabla}^{h-v}(h X, v Y)=-2 h\left(\nabla_{h X} v Y\right) \\
B_{\nabla}^{h-v}(v X, h Y)=-2 v\left(\nabla_{v X} h Y\right)
\end{array}\right.
$$

and then we have the conclusion.

Let us recall the well-known fundamental tensor fields of O'Neill-Gray:

$$
\left\{\begin{array}{l}
T(X, Y)=h\left(\nabla_{v X} v Y\right)+v\left(\nabla_{v X} h Y\right) \\
A(X, Y)=v\left(\nabla_{h X} h Y\right)+h\left(\nabla_{h X} v Y\right) .
\end{array}\right.
$$

Then, a comparison of last two equations yields

$$
\left\{\begin{array}{l}
C_{\nabla}^{h-v}(X, Y)=2[T(X, v Y)+A(X, h Y)] \\
B_{\nabla}^{h-v}(X, Y)=-2[T(X, h Y)+A(X, v Y)]
\end{array}\right.
$$

a fact which justifies the second name of $T$ and $A$ as invariants of (2.1) 6 , p. 9]. 


\section{Generalized product conjugate connections}

In this section we present a natural generalization of the product conjugate connection.

Definition 3.1 A generalized product conjugate connection of $\nabla$ is

$$
\nabla^{(E, C)}=\nabla^{(E)}+C
$$

with $C \in T_{2}^{1}(M)$ arbitrary.

Since the duality $\nabla \leftrightarrow \nabla^{(E)}$ is a main feature of $\nabla^{(E)}$, let us search for tensor fields $C$ such that $\left(\nabla^{(E, C)}\right)^{(E, C)}=\nabla$. From

$$
\left(\nabla^{(E, C)}\right)_{X}^{(E, C)} Y=\nabla_{X} Y+E(C(X, E Y))+C(X, Y)
$$

it results that we are interested in finding solutions $C$ to

$$
E(C(X, E Y))+C(X, Y)=0 .
$$

Let us remark that:

i) $C_{0}=\nabla E$ is a particular solution of (3.3),

ii) if $C$ is a solution, then $E \circ C$ is also a solution.

So, let us search the duality property for

$$
\nabla^{(E, \lambda, \mu)}=\nabla^{(E)}+\lambda \nabla E+\mu E(\nabla E)=(1+\mu) \nabla^{(E)}+\lambda \nabla E
$$

with $\lambda$ and $\mu \in \mathbb{R}$.

Proposition 3.2 The duality $\nabla \leftrightarrow \nabla^{(E, \lambda, \mu)}$ holds only for the pairs $(\lambda, \mu) \in\{(0,0),(0,-2),(1,-1),(-1,-1)\}$.

\section{Proof From}

$$
\left(\nabla^{(E, \lambda, \mu}\right)_{X}^{(E, \lambda, \mu)} Y=\left[(1+\mu)^{2}+\lambda^{2}\right] \nabla_{X} Y+2 \lambda(1+\mu) E\left(\nabla_{X} Y\right)
$$

it results the system

$$
\left\{\begin{array}{l}
(1+\mu)^{2}+\lambda^{2}=1 \\
\lambda(1+\mu)=0 .
\end{array}\right.
$$

which has the above solutions.

Let us point out that

$$
\left\{\begin{array}{l}
\nabla^{(E, 0,0)}=\nabla^{(E)} \\
\nabla^{(E, 0,-2)}=-\nabla^{(E)} \\
\nabla^{(E, 1,-1)}=\nabla \\
\nabla^{(E,-1,-1)}=-\nabla
\end{array}\right.
$$


which confirm our result.

Returning to the general case (3.1), let us present the generalizations of some relations from Proposition 1.1:

1. $\nabla^{(E, C)} E=-\nabla E+C(\cdot, E \cdot)-E \circ C$. Then $\nabla \in \mathcal{C}_{E}(M)$ if and only if $\nabla^{(E, \lambda \nabla E+\mu E \circ \nabla E)} \in \mathcal{C}_{E}(M)$ with $\lambda$ and $\mu$ arbitrary real numbers.

2. the discussion above.

3. $T_{\nabla^{(E, C)}}=T_{\nabla}+E\left(d^{\nabla} E\right)+2 C_{\text {skew }}$, where $C_{\text {skew }}$ is the skew-symmetric part of $C$, i.e. $2 C_{\text {skew }}(X, Y)=C(X, Y)-C(Y, X)$. So, if $C$ is symmetric and $\nabla \in \mathcal{C}_{E}(M)$, then $\nabla$ and $\nabla^{(E, C)}$ have the same torsion.

4. $R_{\nabla^{(E, C)}}(X, Y) Z=E\left(R_{\nabla}(X, Y) E Z\right)+C\left(X, E\left(\nabla_{Y} E Z\right)\right)-C\left(Y, E\left(\nabla_{X} E Z\right)\right)-$ $C([X, Y], Z)+E\left(\nabla_{Y} E(C(Y, Z))\right)-E\left(\nabla_{Y} E(C(X, Z))\right)$.

Acknowledgement. The first author acknowledges the support by the research grant PN-II-ID-PCE-2011-3-0921.

\section{References}

[1] A. Bejancu, H. R. Farran, Foliations and geometric structures, Mathematics and Its Applications 580, Springer, Dordrecht, 2006. MR2190039 (2006j:53034)

[2] A. Bejancu, H. R. Farran, Riemannian Metrics on the Tangent Bundle of a Finsler Submanifold, An. Ştiinţ. Univ. Al. I. Cuza Iaşi. Mat., 56(2010), no. 2, 429-436. DOI 10.2478/v10157-010-0030-8

[3] F. Bullo, A. D. Lewis, Geometric control of mechanical systems. Modeling, analysis, and design for simple mechanical control systems, Texts in Applied Mathematics, 49, Springer-Verlag, New York, 2005. MR2099139 (2005h:70030)

[4] O. Calin, H. Matsuzoe, J. Zhang, Generalizations of conjugate connections, Proceedings of 9th International Workshop on Complex Structures, Integrability, and Vector Fields, 2009, 26-34.

[5] V. Cruceanu, Almost hyperproduct structures on manifolds, An. Ştiinţ. Univ. Al. I. Cuza Iaşi Mat., 48(2002), no. 2, 337-354. MR2007448 (2004h:53040)

[6] M. Falcitelli, S. Ianuş, A. M. Pastore, Riemannian submersions and related topics, World Scientific Publishing Co., Inc., River Edge, NJ, 2004. MR2110043 (2005k:53036) 
[7] V. F. Kirichenko, Method of generalized Hermitian geometry in the theory of almost contact manifold, Itogi Nauki i Tekhniki, Problems of geometry, 18(1986), 25-71; translated in J. Soviet. Math., 42(1988), no.5, 1885-1919. MR0895367 (88g:53042)

Department of Mathematics and Computer Science

West University of Timişoara

Bld. V. Pârvan nr. 4, 300223 Timişoara

România

adara@math.uvt.ro

Faculty of Mathematics

University "Al. I. Cuza"

Iaşi, 700506

România

mcrasm@uaic.ro

http://www.math.uaic.ro/ morasm 